\title{
A Genetic Programming-based Approach for Classifying Pancreatic Adenocarcinoma: the SICED Experience
}

Gianni D'Angelo ( $\sim$ giadangelo@unisa.it )

University of Salerno: Universita degli Studi di Salerno

Maria Nunzia Scoppettuolo

University of Salerno: Universita degli Studi di Salerno

Anna Lisa Cammarota

University of Salerno: Universita degli Studi di Salerno

Alessandra Rosati

University of Salerno: Universita degli Studi di Salerno

Francesco Palmieri

University of Salerno: Universita degli Studi di Salerno

\section{Research Article}

Keywords: Ductal adenocarcinoma, Pancreas, Genetic Programming, Machine Learning.

Posted Date: March 7th, 2022

DOI: https://doi.org/10.21203/rs.3.rs-1273431/v1

License: (9) This work is licensed under a Creative Commons Attribution 4.0 International License.

Read Full License 


\title{
A Genetic Programming-based Approach for Classifying Pancreatic Adenocarcinoma: the SICED experience
}

\section{Gianni D'Angelo ${ }^{1^{*}}$, Maria Nunzia Scoppettuolo ${ }^{2}$, Anna Lisa Cammarota $^{2}$, Alessandra Rosati ${ }^{2}$ and Francesco Palmieri ${ }^{1}$ \\ ${ }^{*}$ Department of Computer Science, University of Salerno, Via} Giovanni Paolo II, 132, Fisciano (SA), 84084, Italy.

${ }^{2}$ Department of Medicine, Surgery and Dentistry "Schola Medica Salernitana", University of Salerno, Via Giovanni Paolo II, 132, Fisciano (SA), 84084, Italy.

\begin{abstract}
Ductal adenocarcinoma of the pancreas is a cancer with a high mortality rate. Among the main reasons for this baleful prognosis is that, in most patients, this neoplasm is diagnosed at a too advanced stage. Clinical oncology research is now particularly focused on decoding the cancer molecular onset by understanding the complex biological architecture of tumor cell proliferation. In this direction, Machine-Learning has proved to be a valid solution in many sectors of the biomedical field, thanks to its ability to mine useful knowledge by biological and genetic data. Since the major risk factor is represented by genetic predisposition, the aim of this study is to find a mathematical model describing the complex relationship existing between genetic mutations of the involved genes and the onset of the disease. To this end, an approach based on evolutionary algorithms (Evolutionary Algorithm) is proposed. In particular, Genetic Programming is used, which allows solving a Symbolic Regression problem through the use of Genetic Algorithms. The identification of these correlations is a typical objective of the diagnostic approach, and is one of the most critical and complex activities in the presence of large amounts of data that are difficult to correlate through traditional
\end{abstract}




\section{Introduction}

Pancreatic cancer (PC) is the fourth most common cause of cancer death in the United States with more than 38,000 deaths in 2013 [1] and is projected to become the second most common cause by 2030 [2]. The situation in Europe is similar in both women and men, with nearly one million deaths for year and reduced quality of life in affected people [3]. In the future, PC will overtake breast cancer as the third leading cause of cancer death [4]. Pancreatic cancer is a complex malignant tumor with a poor prognosis since it does not show particular signs at an early stage, and even when they are present, they are rather vague disorders, which can be misinterpreted. For these reasons, the diagnosis is often made when the disease is already extensive.

Clearer symptoms (which vary depending on where cancer began in the pancreas) appear when cancer has started to spread to nearby organs or has blocked the bile ducts.

However, as the size of the tumor increases, the symptoms also tend to become more noticeable. They depend on many factors, including the type and location of the tumor, as well as on the impact it has on the body in general. They can result in loss of weight and appetite, jaundice, pain in the upper abdomen or back, a feeling of bloating or fullness, weakness, nausea or vomiting.

Other fairly common symptoms include intestinal problems, diabetes, indigestion, heartburn, nausea and vomiting, fever and chills, extreme fatigue, acute pancreatitis of unexplained origin, bulky, clear and foul-smelling stools.

It is therefore diagnosed at an advanced stage of its natural history and is resistant to chemo- and radiotherapy [5]. Surgical removal is the only potentially curative treatment and about $80 \%-85 \%$ of cancers are not surgically removed at the time of diagnosis. However, accurate screening of patients at high risk of developing PC could allow an early diagnosis, when surgical removal of the tumor is still possible. Therefore, identifying high-risk population is crucial, as well as understanding the risk factors for developing PC. Several factors (environmental and genetic) have been identified as being responsible for the increased risk of developing pancreatic cancer [13], such as age, smoking [14,15], alimentation [16], alcoholic beverages [17], chronic pancreatitis [18], diet and obesity [20], diabetes [21], genetic diseases, environmental pollution, and more. 
About $70 \%$ of pancreatic cancers originate from the head of the organ and most of these develop from the ducts that carry digestive enzymes (pancreatic ductal adenocarcinoma- PDAC). Despite recent advances in surgical techniques and medical therapies, the survival on average for a $\mathrm{PC}$ patient is approximately 4-6 months from the diagnosis [6], while the survival rate on average at 5-year is $; 8 \%$ [7]. Pancreatic mucinous cystic neoplasm (MCN) accounts for 2-5\% of all exocrine pancreas neoplasms and is characterized instead by a far better prognosis after surgical eradication. MCN is a cysticforming, mucin-producing neoplasm and a correct clinical approach is often hampered by the difficult differential diagnosis in respect to intraductal papillary mucinous neoplasms (IPMN) or oligocystic serous adenomas before surgery $[8,9]$. Of note, both IPMNs and MCNs can shift towards a more aggressive form [10].

In this complex picture, in pancreatic neoplasms, the most common and early events that allow cells to acquire genetic instability are the dysfunction and critical shortening of telomeres that lead to impairment of chromosomal integrity. This phenomenon is traceable both in precancerous lesions and in invasive carcinomas $[11,12]$. Pancreatic cancer is therefore a disease caused by the accumulation of mutations. As an example, one of the first genetic analysis in 24 patients carrying pancreatic adenocarcinomas, showed that, the genetic basis of the neoplasm is complex and heterogeneous. In each tumor, an average of 63 genetic mutations (mainly of a single nucleotide substitution) have been identified, which are organized into twelve signal transduction pathways, whose alterations are fundamental in the oncogenetic process. Not all tumors exhibit alterations in all pathways, and key mutations in each pathway appear to differ from tumor to tumor [12].

The main alterations include:

1. mutations activating the KRAS oncogene in $90 \%$ of cases;

2. mutations inactivating the p16 oncosuppressor encoded by the CDKN2A gene in almost all cases;

3. mutations inactivating the TP53 gene, encoding the oncosuppressor p53 in $60 \%$ of cases;

4. deletion of the SMAD4 DPC4 gene in $50 \%$ of cases.

Precisely because of the high genetic heterogeneity of pancreatic cancer, there is still no genetic test to accurately define the lesions. For this reason, the identification of mutations is the path that can lead to the development of reliable diagnostic tests to guarantee patients an earlier diagnosis, hopefully before the tumor manifests itself clinically. The identification of the mutations associated with the main lesions and pre-invasive forms and the description of their role in the evolution of the tumor cell can provide a fundamental contribution in the perspective of developing a rapid and accurate diagnostic system and is the first step for understanding the mechanisms of pancreatic tumorigenesis. 


\section{Materials and Methods}

\subsection{Dataset}

To better characterize and understand the genetics of pancreatic cancer, we have analyzed the overall picture of the genetic alterations that underlie the heterogeneity of pancreatic adenocarcinoma. To this end, we have used several clinical and biological databases in order to develop innovative approaches in the early diagnosis of pancreatic cancer.

Thanks to the study called Pan-Cancer Project which lasted about 10 years, which involved 16 working groups of the International Cancer Genome Consortium (ICGC), and the US project called The Cancer Genome Atlas (TCGA), the mapping of over 2,600 genomes belonging to 38 different types of cancer has been carried out. This has made it possible to create a huge database that allows the study of multiple aspects of the development, cause, progression and classification of cancer. The information gathered by these projects has been made public in order to accelerate the understanding of 
the molecular basis of cancer through the new generation of genome analysis technologies.

In our study, we have used three different databases available at the following addresses, respectively:

- https://portal.gdc.cancer.gov/

- https://cbioportal.org

- https://dcc.icgc.org

They include histological images, various information on patient history, clinical data and all genetic information, including point mutations and Copy Number Variants $(\mathrm{CNV})$. The three databases include 1067 pancreatic cancer patients, and they have been got by 5 projects, that are:

1. PAAD-US: 185 patients coming from United States with ductal adenocarcinoma (https://dcc.icgc.org/projects/PAAD-US);

2. PACA-CA: 317 patients coming from Canada with ductal adenocarcinoma (https://dcc.icgc.org/projects/PACA-CA);

3. PACA-AU: 461 patients coming from Australia with ductal adenocarcinoma (https://dcc.icgc.org/projects/PACA-AU);

4. PAEN-AU: 67 patients coming from Australia with pancreatic neuroendocrine tumor (https://dcc.icgc.org/projects/PAEN-AU);

5. PAEN-IT: 37 patients coming from Italy with entero-pancreatic endocrine tumor and rare exocrine pancreatic tumors (https://dcc.icgc.org/projects/ PAEN-IT).

Due to the large number of risk factors involved in the pathogenesis of pancreatic adenocarcinoma, for each of these patients we have also extrapolated demographic data, genetic data, and risk factors. More specifically, the following factors have been considered.

\section{Demographic:}

- Age (more than $80 \%$ of adenocarcinomas affect people aged between 60 and 80 years);

- Vital state (patient dead or still alive at the time of genetic analysis);

- Age at diagnosis of pancreatic cancer.

\section{Genetic:}

- History of Acute Pancreatitis;

- Medical history Diabetes mellitus;

- Family history of cancer (pancreas or other cancer);

- Genetic mutations (point, small insertions and small deletions);

- CNV (Copy Number Variants).

\section{Cancer characteristic:}

- Type of pancreatic cancer:

- Cystic, Mucinous and Serous Neoplasms; 
- Adenomas and Adenocarcinomas;

- Ductal and Lobular Neoplasms;

- Epithelial Neoplasms, NOS.

- Tumor stage:

- Stage IA. The tumor is limited to the pancreas site only and in its maximum extension measures less than $2 \mathrm{~cm}$ (localized - resectable);

- Stage IB. The tumor is limited to the pancreas and measures more than $2 \mathrm{~cm}$ (localized - resectable);

- Stage IIA. The cancer extends outside the pancreas but does not involve major local arteries or lymph nodes (locally advanced - resectable or borderline resectable);

- Stage IIB. The tumor may or may not extend outside the pancreas but does not involve the major arteries. Local lymph nodes are involved (locally advanced - resectable or borderline resectable);

- Stage III. The tumor involves important local arteries. Local lymph nodes may be involved (locally advanced - unresectable);

- Stage IV. The tumor can be of any size. The tumor has spread to other organs (metastatic - unresectable).

- Tumor grade:

- G1: well differentiated tumor;

- G2: moderately differentiated tumor;

- G3: poorly differentiated tumor;

- G4: undifferentiated tumor.

Note that the tumor grade allows us to classify the differentiation status of the cells in order to define the degree of aggressiveness of the tumor.

\section{Other:}

- Cigarette smoking (the only environmental factor definitely correlated with the risk of pancreatic adenocarcinoma);

- Alcohol consumption (data divided by frequency and intensity);

- Survival from initial diagnosis (expressed in days).

Since one of the major risk factors is represented by genetic predisposition, we preferred to focus our attention on genes mutated in high percentages and, therefore, more frequent in patients with pancreatic cancer. Furthermore, among the different somatic mutations collected in our 3 databases, we detected a high number of point mutations. Most of mutations that we found are the following: synonym, missense, nonsense, small insertions and deletions (frameshift and in frame), mutations in splicing sites or in untranslated regions (UTR).

Below are the genes used in our analysis: KRAS, Tp53, CDKN2A, TGF-1 $\beta$, Smad genes, BRCA1, BRCA2, PRSS1/CFTR, APC, BAG3, STK11/LKB1, MLH1, MSH2, MSH6, PMS2, ATM, PALB2, TTN, DNM1P47, HDAC5, 
EGF/ERBB2, STAT3, PTEN, KLF4, PIK3CA, BRAF, ARID1A, ROBO2, KDM6A, PREX2, CDK6, GNAS, ATRX, KMT2D.

\subsection{Features Selection}

Different types of mutations have been considered for each of the aforementioned genes. This led to a total number of parameters (i.e. characteristics) equal to 471 .

As it is known, the choice of highly informative and independent discriminating features is a crucial step to obtain an efficient pattern recognition, classification or regression algorithm [19]. Besides, the number of used features should be kept low in order to obtain high performance.

Consequently, a typical preliminary step in many Machine-Learning applications consists in the selection of the features (Features Selection), or, more generally, in the reduction of the dimensionality of the so-called input space.

In our study, it can be seen that the available dataset includes, for each patient, a very high number of features for each gene (i.e. 471). The extraction of information from a dataset characterized by such a large number of features would require many training examples (patients). Unfortunately, as described in the previous section, the dataset includes only 1067 cases, which could negatively affect the performance of the proposed system.

For this reason, in order to evaluate the actual usefulness of all the features, the link between these features and the output was evaluated through WEKA's attribute selection techniques. In particular, two methods were used, namely: GainRatioAttributeEval and InfoGain. Note that, as better described in the next sub-section, our goal is to classify the cancer stage.

The results obtained for both algorithms suggested that only the features f111, f197, f279, f288, f318, f352, and $\mathrm{f} 443$ are relevant for the classification. Below is the correspondence between these features and their meaning:

- $\mathrm{f} 111 \rightarrow$ TP53 chr17: g.7674226A>C Replacement Missense M246R

- $\mathrm{f} 197 \rightarrow$ TTN chr2: g.17 8528652 del TCTGAGAG deletion inframe Deletion E35697_S35699del

- $\mathrm{f} 279 \rightarrow$ DNM1P47 chr15: g.101752915C >A Replacement Non Coding Transcript Exon DNM1P47

- $\mathrm{f} 288 \rightarrow$ DNM1P47 chr15: g.101754458G $>$ C Replacement Non Coding Transcript Exon DNM1P47

- $\mathrm{f} 318 \rightarrow$ ATM chr11: g.108287646T>G Replacement Stop Gained L1347*

- $\mathrm{f} 352 \rightarrow$ APC chr5: g.112841068A > G Replacement Missense D1825G

- $\mathrm{f} 443 \rightarrow$ GNAS chr20: g.58909366G > A Replacement Missense R844H

\subsection{Genetic programming-based tumor classification}

As previously mentioned, the aim of this study is to classify the type of pancreatic neoplasm. More specifically, we are interested in finding the relation existing among gene mutations and cancer stage. We remark that a classifier can be seen as a function that takes a features vector as input and assigns a 
class label to it. Accordingly, in our study, we considered a 471-dimensional vector as input and two classes as output, one corresponding to the presence of MCN type (Class 1) and the other corresponding to PDAC type (Class 0).

In order to build such a classifier, in this study, an Evolutionary-based Algorithm is proposed. More specifically, the Genetic Algorithm (GA) is used to perform a Symbolic Regression (SR) analysis.

We remark that regression is used to mine insights contained in data, and Symbolic Regression is a method widely known and used in the field of regression analysis. It is used to discover a mathematical formula describing an existing relation between multiple input/output pairs of variables [20].

In recent decades, advances in the field of Evolutionary Algorithms (EAs) have allowed the spread of SRs.

The main goal of an EA is to provide solutions to many types of real-world problems by using Darwin's theory of evolution. More specifically, EA makes usage of a population of solutions, each called individual, which are combined through the operators of selection, crossover, and mutation to create a new population of solutions until the best individual (i.e. the best solution) in the population is found-out.

In order to solve a SR problem Jhon Koza [21] used GA, by laying the foundations of the Genetic Programming (GP) [22].

Basically, GP is an SR running by EAs. Like in AEs, in GPs there are numerous individuals which correspond to a different mathematical formula. Each formula, and then individual, is represented by a tree structure (see Fig. 1 ), whose leaves represent the operands of the formula, while the mathematical operations $\left(+,-,^{*}, /\right.$, etc.) are represented by the remaining nodes.

A fitness function is used to evaluate the goodness of a solution. Generally, it estimates the error between the actual and expected result for all the entries of the dataset provided as input.

Mathematical Model

Fig. 1 Tree representation of a mathematical formula for GP-based elaboration. 
The algorithm evolves in a similar way to the GA principles, that is through the operations of selection, crossover, and mutation. In the selection phase, the fitness function guides the choice of the best individuals corresponding to the formulas that express a result closest to the real one. These individuals are combined through the crossover operation (also called recombination), which exchanges some sub-trees (genes) of two individuals (parents). As shown in Figure 2, the resulting new individual (offspring) includes the best genetic information of two parents, i.e. the offspring is made up of pieces of functions belonging to the best solutions.

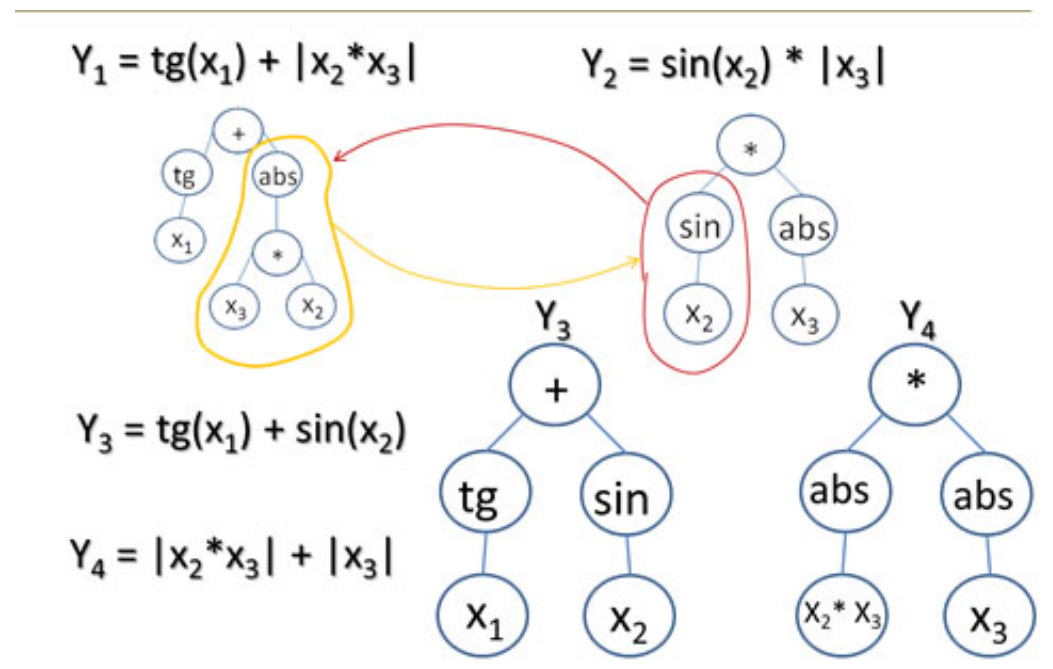

Fig. 2 Crossover operation between two formulas.

Depending on the data structure used to store genetic information, the crossover can be performed differently [23, 24]. In order to ensure genetic diversity, the mutation operation is carried out on a number of new individuals. The mutation alters one or more genes (parts of functions) based on a userdefined probability of mutation. The set of new resulting individuals represents the new population, which is used in the next cycle of evolution. The cycle is repeated until the fitness function reaches the desired value.

Figure 3 shows a sketch of the operations described. As can be seen, the system employs a supervised learning approach, in fact both the input and the output are provided as data to the algorithm. The output is a mathematical formula that roughly represents the relationship between the inputs and the corresponding outputs provided. 


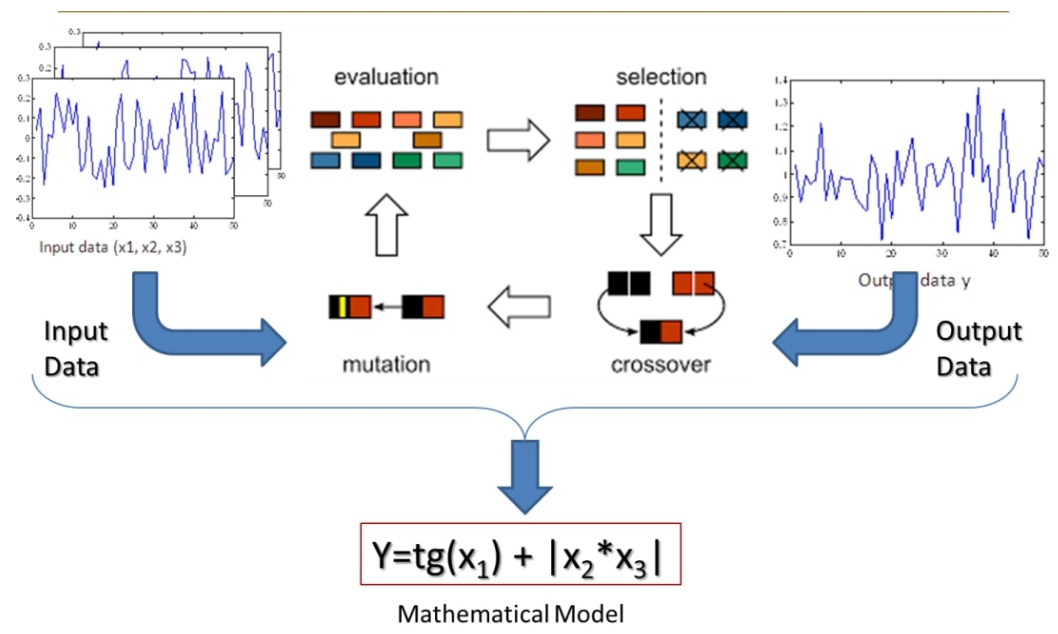

Fig. 3 A sketch of the Genetic Programming.

Nevertheless, GP cannot be used directly to implement a classifier, because GP acts on numeric values for both input and output, while the classification lays on categorical variables for the output.

This leads to the need to make some assumptions to implement a classifier with GP. Accordingly, the class values have been changed to take on numeric values. In particular, we have assigned the numerical value 1 to the PDAC type (Class 0 ), while 0 to the MCN type (Class 1 ). In this way, GP will try to find a formula that fits all numeric inputs (vector of features) to the corresponding numeric output ( 1 or 0$)$ of the data set.

As it might expect, for a given vector of input features, the outcome of the extracted formulas with an high probability will never exactly match the corresponding numeric output class ( 1 or 0 ).

To solve this problem, the concept of threshold has been introduced. The threshold, which ranges from 0 to 1 , is chosen as the point of maximum separation between the results obtained for all items in the dataset. For a given vector of features, if the result of the formula is greater than or equal to this threshold, then that vector belongs to Class 1, otherwise it belongs to Class 0 .

In order to give an idea of this approach, in Figure 4 an example is shown. As depicted, both actual and expected results are shown for each entry of the dataset. Note that, for the sake of clarity, all results have been grouped by class. As shown, in this case, the best threshold is 0.46 . As a consequence, all the outcomes above this threshold are considered to belong to Class 1, while the others to Class 0 . 


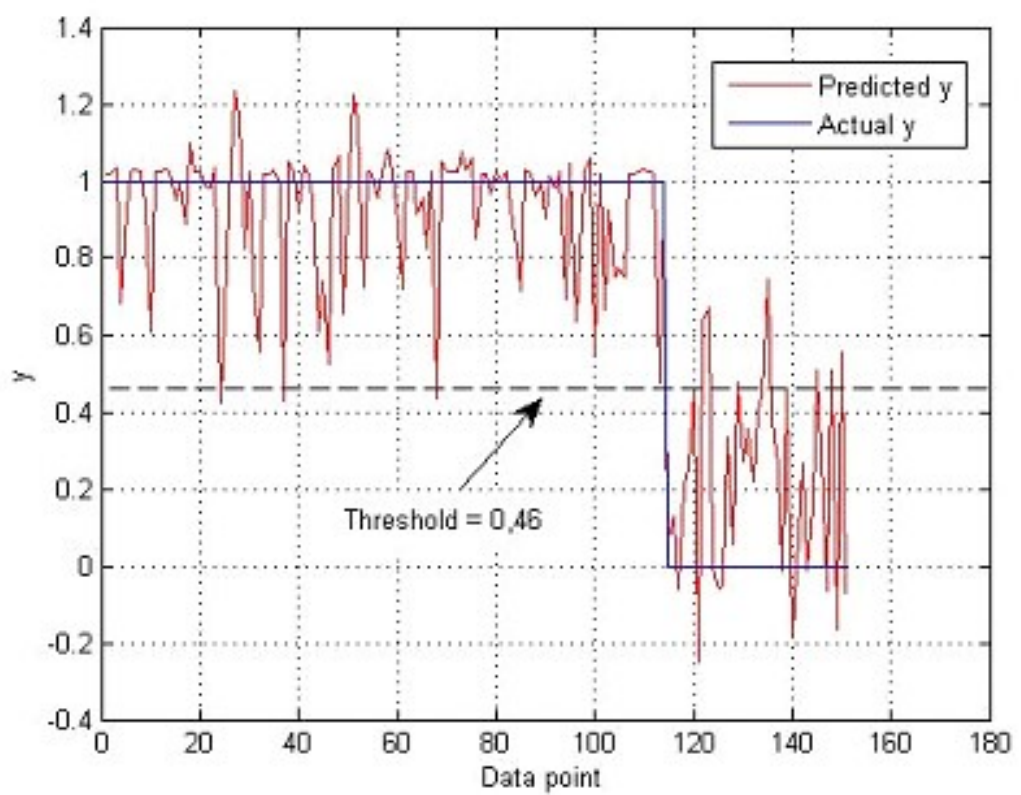

Fig. 4 Threshold example. The best separation between the predicted data (red) and the real data (blue) is identified by the threshold value equal to 0.46 .

\section{Experiments and Results}

\subsection{Experiments Flow}

Figure 5 shows a flow diagram of the implementation of the experiments carried out. As can be seen, the dataset is divided into two families: the Training and the Testing set. The former is used to create a model, while the latter is used to validate the reliability of the extracted model. In order to avoid the phenomenon of biasing, the 5-fold cross-validation was used [25], and therefore, the scheme shown in Figure 5 was repeated 5 times, each for each folder of the cross-validation. More specifically, the overall dataset was divided into 5 disjoint subsets (named folds), each including approximately the same number of instances. In each experiment, a single fold is used as the testing set, while the aggregation of the remaining ones is used as the training set. The outcomes of each experiment are averaged to provide a single performance evaluation result.

The reliability of the outcomes is evaluated by metrics derived from the confusion matrix associated with a binary classifier, as described in the following. 
Fig. 5 Experiments Flow Diagram. It is repeated 5 times in according with the 5 -fold cross-validation approach.

\subsection{Evaluation metrics}

In a binary classifier, data associated with the two classes are referred to as positive and negative instances, respectively. In our case, we identified the Class 1 as positive, whereas Class 0 as negative. The instances that are correctly classified as positive and negative are called true positive (TP) and true negative (TN), respectively. While the positive instances that are incorrectly classified as negative are called false negatives (FN). Finally, negative instances that are incorrectly classified as positive are called false positives (FP). As a consequence, the overall number of positives and negatives can be represented by $\mathrm{P}=(\mathrm{TP}+\mathrm{FN})$ and $\mathrm{N}=(\mathrm{TN}+\mathrm{FP})$, respectively. Their representation in a table is known as confusion matrix.

Several metrics can be derived by the confusion matrix [26]. In our study, we used the following:

- Accuracy: correctly classified portion of data. It is given by:

$$
A c c=\frac{T P+T N}{T P+T N+F P+F N}
$$

- Sensitivity: also known as TPR (True Positive Rate) portion of positives that are correctly identified as such.

$$
T P R=\frac{T P}{T P+F N}
$$


- Specificity: also known as TNR (True Negative Rate) portion of negatives that are correctly identified as such.

$$
T N R=\frac{T N}{T N+F P}
$$

- Precision: It measures how many positives have been correctly identified against all instances classified as positive.

$$
\text { Prec }=\frac{T P}{T P+F P}
$$

- F-Measure: It is the harmonic average of Precision and Sensitivity.

$$
F_{-} \text {Meas }=\frac{2 * T P R * \text { Prec }}{T P R+\text { Prec }}
$$

- $A U C$ (Area Under Curve): It measures the surface subtended by the ROC curve. It is an estimate of how much greater is the probability that a classifier will be able to rank a randomly chosen positive instance over a randomly chosen negative one.

$$
A U C=\frac{T P R+T N R}{2}
$$

\subsection{Implementation details}

The GP-based classifier was implemented in the MATLAB environment using an open-source library for symbolic data mining, namely the GPTIPS library (Genetic Programming Toolbox for the Identification of Physical Systems) [27]. It uses a multi-gene-based genetic programming (MGGP) as the main engine, which is a variant of the GP approach for formula discovery. In particular, in MGGP a formula is seen as a single gene of an individual, while the individual is represented by a weighted linear combination of these genes plus a bias (offset). Optimal values for weights are automatically obtained using the leastsquares approach to fit the expected result to the actual output data as closely as possible. This individual representation is also known as symbolic scaling regression, which differs from the native SR in the presence of bias and linear combination. The linear combination of genes can capture nonlinear behavior much more effectively than pure SR.

Using MATLAB as a core platform confers to this library the advantage of having a pluggable architecture, which means that users can easily change the algorithm setting parameters and fitness function by including their own code as an add-on in the GPTIPS library. Among the large number of configuration options that can be set, there are typical ones, such as population size, maximum number of generations, number of genes included in an individual, tournament size, and mutation size.

Once these setup parameters have been specified by the user, the algorithm evolves as follows. First, a population is randomly generated. Each individual 
can contain a random number of genes between 1 and the user-specified Gmax value, and a tree depth level can also be specified. It is important to point out that even though a high $G \max$ can capture many hidden non-linearity behaviors in the data, it can also lead to the problem of overfitting, i.e. the output formula fits well only to the training set, but not to unknown data. Furthermore, in this phase, the algorithm tries to guarantee the diversity between the generated individuals and between the genes belonging to the same individual. This is essential to avoid duplications, which can negatively influence the evolution of the algorithm. As in the standard GP, individuals are probabilistically selected for the crossover operation at each generation. There are two types of crossover operations, namely the low and high level crossover. In the first level, the first two individuals with a higher fitness score are chosen as parents, then a gene is chosen at random from each parent. These genes are exchanged between parents to create two children. These descendants are then used in the new population. On the other hand, the latter allows parents to swap more than one gene, i.e. they can swap entire trees, compatibly with the respective Gmax value. However, if the exchange leads to a number of genes greater than the Gmax constraint, some genes are randomly pruned. In this way, people with better fitness scores are expected to survive and evolve in the learning process.

\subsubsection{Experimental setup}

Below, the parameters used in all the experiments:

- Initialization: a population of 100 individuals was used. Each individual can include up to five levels for the depth of the tree, a Gmax $=5$, and can include the following arithmetic operators $\mathrm{F}=+,-,{ }^{*}, /$, sqrt, exp. The bias ranges from -10 to 10 .

- Fitness function: the fitness function provides a measure of the performance of a specific individual to solve the problem and guides the evolutionary process towards the best solution. One possible metric is to measure the error between the original and expected output of a solution. Therefore, the root mean square (RMS) of the errors calculated for the entire data set is used as a fitness function.

- Crossover Operation: A low-level crossover was used. To select individuals for crossover, the tournament scheme was used. The fitness function defined above was used for parent selection. The tournament size was fixed to 3 .

- Mutation: the mutation operation can heavily influence the results, as the mutation swaps a subtree with a new one generated randomly. If the point of exchange is a terminal node, the resulting gene only mutates slightly and the mutation does not severely affect the tree. On the other hand, a point that falls on functional nodes can cause a large variation in the tree. For these reasons, a mutation probability of zero was chosen.

- Termination: the above steps are applied recursively in order to refine the solutions during the evolution process. This iterative process is stopped when 


\subsection{Results}

In order to evaluate the performance of the proposed approach, the whole dataset was firstly divided into two subsets, namely evaluation and testing dataset, respectively. The former, used to train, evaluate and compare the proposal with the state-of-the-art, includes data coming from PAAD-US, PACA-CA, and PAEN-IT projects containing 5 positives and 497 negatives. The latter, used to test the proposed mathematical formula, includes data coming from PACA-AU project containing 432 negatives and 6 positives.

According to the aforementioned k-fold cross-validation technique, the evaluation dataset was randomly divided into 5 disjoint subsets and the proposed classifier was trained on each training set obtained, while its effectiveness was verified on the corresponding test set.

As can be expected, since GP is based on a heuristic approach, the formulas provided in the different tests can be very different even if the algorithm is applied on the same training set. Therefore, for each test, we repeated the experiment 20 times.

Table 1 shows the performance of the resulting best formulas. In order to demonstrate the significance of the results, the mean value, standard deviation and confidence interval (at 95\% confidence level) for all metrics are also reported.

As illustrated, the proposed GP-based classifier obtained a high score for all metrics, which confirms its effectiveness in classifying the type of tumor starting from the considered genes. In particular, it can be seen that there are only a few FPs and FNs, which leads to very high scores for the metrics. Furthermore, the higher score for AUC (0.93 on average) confirms the ability of the proposed classifier to perform better than a random classifier.

Table 1 Experimental results obtained by the 5-fold cross validation technique.

\begin{tabular}{|c|c|c|c|c|c|c|c|c|c|c|}
\hline \multirow[b]{2}{*}{ Test } & \multicolumn{4}{|c|}{ Confusion matrix } & \multirow[b]{2}{*}{ Acc } & \multirow[b]{2}{*}{ TPR } & \multirow[b]{2}{*}{ TNR } & \multirow[b]{2}{*}{ Prec } & \multirow[b]{2}{*}{ FMeas } & \multirow[b]{2}{*}{ AUC } \\
\hline & $\mathbf{T P}$ & FP & FN & $\mathbf{T N}$ & & & & & & \\
\hline 1 & 1 & 14 & 0 & 85 & 0.86 & 1.00 & 0.86 & 0.07 & 0.13 & 0.93 \\
\hline 2 & 1 & 0 & 0 & 99 & 1.00 & 1.00 & 1.00 & 1.00 & 1.00 & 1.00 \\
\hline 3 & 1 & 0 & 1 & 98 & 0.99 & 0.50 & 1.00 & 1.00 & 0.67 & 0.75 \\
\hline 4 & 1 & 10 & 0 & 89 & 0.90 & 1.00 & 0.90 & 0.09 & 0.17 & 0.95 \\
\hline 5 & 1 & 0 & 0 & 99 & 1.00 & 1.00 & 1.00 & 1.00 & 1.00 & 1.00 \\
\hline Mean & & & & & 0.95 & 0.90 & 0.95 & 0.63 & 0.59 & 0.93 \\
\hline StDev & & & & & 0.07 & 0.22 & 0.07 & 0.50 & 0.43 & 0.10 \\
\hline \multirow[t]{2}{*}{ Cin@95 } & & & & & $(0.87$ & $(0.62$ & $(0.87$ & $(0.00$ & $(0.06$ & $(0.80$ \\
\hline & & & & & 1.03) & 1.18) & 1.04) & 1.26) & 1.12) & $1.05)$ \\
\hline
\end{tabular}




\subsection{Comparison}

Below are the results obtained using some of the most common ML techniques available in the literature, such as Multi Layer Perceptron (MLP), Naive Bayes (NB), and Decision Tree (J48). All reported results were obtained by using the same folds of the previous experiments. In Table 2, the metrics averaged on all folds are only reported.

As depicted, even though the Accuracy achieved excellent values (0.99 on average), Sensitivities were very low (0.06 on average). This means that such techniques are not able to correctly distinguish the two classes, and, further, they classify all entries as negative. Indeed, the Specificity is very high (0.99 on average).

Table 2 Averaged results of the 5-fold cross validation obtained by state-of-the-art techniques.

\begin{tabular}{|c|c|c|c|c|c|c|c|c|c|c|}
\hline \multirow[b]{2}{*}{ Method } & \multicolumn{4}{|c|}{ Confusion matrix } & \multirow[b]{2}{*}{ Acc } & \multirow[b]{2}{*}{ TPR } & \multirow[b]{2}{*}{ TNR } & \multirow[b]{2}{*}{ Prec } & \multirow[b]{2}{*}{ FMeas } & \multirow[b]{2}{*}{ AUC } \\
\hline & $\mathbf{T P}$ & FP & FN & TN & & & & & & \\
\hline MLP & 0 & 3 & 5 & 494 & 0.98 & 0.00 & 0.99 & 0.00 & - & 0.50 \\
\hline NB & 1 & 3 & 4 & 494 & 0.99 & 0.20 & 0.99 & 0.25 & 0.22 & 0.60 \\
\hline $\mathrm{J} 48$ & 0 & 0 & 5 & 497 & 0.99 & 0.00 & 1.00 & - & - & 0.50 \\
\hline
\end{tabular}

\subsection{Modeling}

Although the high scores achieved by all metrics in the previous experiments confirm the effectiveness of the proposed classifier, we remark that the potentiality offered from the proposal is to provide a mathematical model expressing the complex relationships existing between cancer typology and genetic mutations, which is extremely desirable in clinical environments.

To this purpose, in the following the formula obtained by using whole dataset is reported.

$$
\begin{gathered}
F=G_{1}+G_{2}-G_{3} \\
G_{1}=0.1332 * f 279-0.06658 * f 111+f 288 \\
G_{2}=0.9334 * f 318+f 352+0.06658 * f 443 \\
G_{3}=0.06658 * E X P(-f 288) *(f 111+f 318)+0.0001388 \\
\text { Tumor Class }=\left\{\begin{array}{l}
1, F \geq 0.01 \\
0, \text { otherwise }
\end{array}\right.
\end{gathered}
$$

As depicted, three genes (Eqs. (8), (9), and (10), respectively) are included in the individual $F$, represented by Equation (7). Finally, the class of the cancer 
is obtained by comparing $F$ with the threshold 0.01, as shown in Equation (11).

The formula emphasizes the complex correlation existing between involved variables and between these variables and the tumor class. Namely, in order to classify the tumor, it is very important to evaluate not only the presence or absence of gene mutations but how they are correlated. Indeed, as it can be deduced by the proposed formula, many combinations of mutations of the involved genes could give the same outcomes (1 or 0$)$.

Finally, in order to prove the accuracy of the proposed formula, we have tested it on the datasets by PACA-AU and PAEN-AU projects including 432 negatives and 6 positives. Table 3 shows the results.

As depicted, all metrics achieved the maximum score, which confirms the effectiveness of the proposal in distinguishing the typology of cancer despite the significant unbalancing between positive and negative samples.

Table 3 Results obtained by testing the proposed formula on data obtained by PACA-AU project.

\begin{tabular}{cccccccccc}
\hline \multicolumn{2}{c}{ Confusion matrix } & & & & & & \\
\cline { 1 - 5 } & & & & & & & & \\
TP & FP & FN & TN & Acc & TPR & TNR & Prec & FMeas & AUC \\
\hline 6 & 2 & 0 & 430 & 1.00 & 1.00 & 0.99 & 0.75 & 0.86 & 1.00 \\
\hline
\end{tabular}

\section{Conclusions}

In this paper, the task of distinguishing the typology of Pancreatic Adenocarcinoma has been addressed by using a Genetic Programming-based approach. The main aim of this work has been to find out a mathematical formula able to express the complex relationship existing between gene mutations and cancer typology. To this end, the problem has been modeled through the usage of Symbolic Regression and solved by a genetic algorithm. Also, the threshold concept has been provided to turn the regression problem into a classification one.

The identification of these correlations is a typical objective of the diagnostic approach, which can be used to improve the prognosis and to provide an early diagnosis.

The formula obtained highlights the complex analysis of a large amount of data, which often is not possible to achieve by traditional statistical techniques.

Although the dataset is definitely unbalanced, the proposed approach has shown to be able to generate high reliability results when evaluated on realworld cases by the cross-validation methodology. Also, comparison with the state-of-the-art has proven the superiority of the proposed approach. 


\section{Declarations}

\section{Authors' contributions}

Conceptualization, G.D.; methodology, G.D.; software, G.D.; validation, G.D.; formal analysis, G.D.; investigation, G.D.; resources, A.R., M.N.S. and A.L.C.; writing - original draft preparation, G.D.; writing - review and editing, G.D., A.R., and F.P.; visualization, G.D.; supervision, G.D. and F.P.; project administration, F.P. and A.R.; funding acquisition, F.P. All authors have read and agreed to the published version of the manuscript'.

\section{Funding}

This research was funded by the Campania Region, Italy through the POR FESR 2014-2020 under the project SICED (System Innovation for Cancer Early Diagnosis), CUP B53D18000150007 - SURF 17061BP000000013.

\section{Institutional Review Board Statement}

The study was conducted according to the guidelines of the Declaration of Helsinki.

\section{Informed Consent Statement}

No explicit informed consent was necessary from all subjects involved in the study because the dataset used are publicly available.

\section{Ethics approval}

Not applicable.

\section{Availability of data and materials}

Publicly available datasets were analyzed in this study. This data can be found here:

- https://portal.gdc.cancer.gov/

- https://cbioportal.org

- https://dcc.icgc.org

\section{Conflict of interest}

The authors declare no conflict of interest.

\section{Consent to participate}

'Not applicable'

\section{Consent for publication}

'Not applicable' 


\section{References}

[1] Siegel, R., Naishadham, D., Jemal, A.: Cancer statistics, 2013. CA Cancer J Clin 63(1), 11-30 (2013)

[2] Rahib, L., Smith, B.D., Aizenberg, R., Rosenzweig, A.B., Fleshman, J.M., Matrisian, L.M.: Projecting cancer incidence and deaths to 2030: the unexpected burden of thyroid, liver, and pancreas cancers in the united states. Cancer Res 74(11), 2913-2921 (2014)

[3] Carrato, A., Falcone, A., Ducreux, M., Valle, J.W., Parnaby, A., Djazouli, K., Alnwick-Allu, K., Hutchings, A., Palaska, C., Parthenaki, I.: A systematic review of the burden of pancreatic cancer in europe: Real-World impact on survival, quality of life and costs. J Gastrointest Cancer 46(3), 201-211 (2015)

[4] Bray, F., Ferlay, J., Soerjomataram, I., Siegel, R.L., Torre, L.A., Jemal, A.: Global cancer statistics 2018: GLOBOCAN estimates of incidence and mortality worldwide for 36 cancers in 185 countries. CA Cancer J Clin 68(6), 394-424 (2018)

[5] Momi, N., Kaur, S., Krishn, S.R., Batra, S.K.: Discovering the route from inflammation to pancreatic cancer. Minerva Gastroenterol Dietol 58(4), 283-297 (2012)

[6] Farthing, M., Roberts, S.E., Samuel, D.G., Williams, J.G., Thorne, K., Morrison-Rees, S., John, A., Akbari, A., Williams, J.C.: Survey of digestive health across europe: Final report. part 1: The burden of gastrointestinal diseases and the organisation and delivery of gastroenterology services across europe. United European Gastroenterology Journal 2(6), 539-543 (2014). https://doi.org/10.1177/2050640614554154

[7] Lucas, A.L., Malvezzi, M., Carioli, G., Negri, E., La Vecchia, C., Boffetta, P., Bosetti, C.: Global trends in pancreatic cancer mortality from 1980 through 2013 and predictions for 2017. Clin Gastroenterol Hepatol 14(10), 1452-14624 (2016)

[8] Scholten, L., van Huijgevoort, N.C.M., van Hooft, J.E., Besselink, M.G., Del Chiaro, M.: Pancreatic cystic neoplasms: Different types, different management, new guidelines. Visc Med 34(3), 173-177 (2018)

[9] Xie, W., Liang, H., Guo, Y., Xiao, S.-Y.: Update on mucinous cystic neoplasm of the pancreas: a narrative review. Journal of Pancreatology 4(3) (2021)

[10] Coriat, R., Pellat, A.: Updates on the treatment of pancreatic diseases: Focus on surgery, electrochemotherapy and rituximab. Journal of Clinical 
[12] Gisselsson, D., Jonson, T., Petersén, A., Strömbeck, B., Dal Cin, P., Höglund, M., Mitelman, F., Mertens, F., Mandahl, N.: Telomere dysfunction triggers extensive DNA fragmentation and evolution of complex chromosome abnormalities in human malignant tumors. Proc Natl Acad Sci U S A 98(22), 12683-12688 (2001)

[13] D'Angelo, G., Palmieri, F.: Enhancing COVID-19 tracking apps with human activity recognition using a deep convolutional neural network and HAR-images. Neural Comput Appl, 1-17 (2021)

[14] D'Angelo, G., Palmieri, F.: Discovering genomic patterns in sars-cov-2 variants. International Journal of Intelligent Systems 35(11), 1680-1698 (2020). https://doi.org/10.1002/int.22268

[15] Elia, S., D’Angelo, G., Palmieri, F., Sorge, R., Massoud, R., Cortese, C., Hardavella, G., De Stefano, A.: A machine learning evolutionary algorithm-based formula to assess tumor markers and predict lung cancer in cytologically negative pleural effusions. Soft Computing 24(10), 7281-7293 (2020). https://doi.org/10.1007/s00500-019-04344-1

[16] D'Angelo, G., Pilla, R., Dean, J.B., Rampone, S.: Toward a soft computing-based correlation between oxygen toxicity seizures and hyperoxic hyperpnea. Soft Comput. 22(7), 2421-2427 (2018). https://doi.org/ 10.1007/s00500-017-2512-z

[17] D'Angelo, G., Rampone, S.: Towards a hpc-oriented parallel implementation of a learning algorithm for bioinformatics applications. BMC Bioinformatics 15(5), 2 (2014). https://doi.org/10.1186/1471-2105-15-S5-S2

[18] D’Angelo, G., Pilla, R., Tascini, C., Rampone, S.: A proposal for distinguishing between bacterial and viral meningitis using genetic programming and decision trees. Soft Computing 23(22), 11775-11791 (2019). https://doi.org/10.1007/s00500-018-03729-y

[19] Visalakshi, S., Radha, V.: A literature review of feature selection techniques and applications: Review of feature selection in data mining. In: 2014 IEEE International Conference on Computational Intelligence and Computing Research, pp. 1-6 (2014). https://doi.org/10.1109/ICCIC. 2014.7238499 
[20] Duffy, J., Engle-Warnick, J.: Using Symbolic Regression to Infer Strategies from Experimental Data, vol. 100. Physica, Heidelberg, ??? (2002). https: //doi.org/10.1007/978-3-7908-1784-3_4

[21] Koza, J.R.: Genetic programming as a means for programming computers by natural selection. Statistics and Computing 4(2), 87-112 (1994). https: //doi.org/10.1007/BF00175355

[22] Affenzeller, M., Winkler, S., Wagner, S., Beham, A.: Genetic Algorithms and Genetic Programming: Modern Concepts and Practical Applications, 1st edn. Chapman-Hall-CRC, ??? (2009)

[23] D'Angelo, G., Palmieri, F.: Gga: A modified genetic algorithm with gradient-based local search for solving constrained optimization problems. Information Sciences 547, 136-162 (2021). https://doi.org/10.1016/j.ins. 2020.08 .040

[24] Hasançebi, O., Erbatur, F.: Evaluation of crossover techniques in genetic algorithm based optimum structural design. Computers \& Structures 78(1), 435-448 (2000). https://doi.org/10.1016/S0045-7949(00)00089-4

[25] Copyright. In: Witten, I.H., Frank, E., Hall, M.A. (eds.) Data Mining: Practical Machine Learning Tools and Techniques (Third Edition), Third edition edn. The Morgan Kaufmann Series in Data Management Systems, p. . Morgan Kaufmann, Boston (2011). https://doi.org/10.1016/B978-0-12-374856-0.00025-0. https: //www.sciencedirect.com/science/article/pii/B9780123748560000250

[26] Davis, J., Goadrich, M.: The relationship between precision-recall and roc curves. In: Proceedings of the 23rd International Conference on Machine Learning. ICML '06, pp. 233-240. Association for Computing Machinery, New York, NY, USA (2006). https://doi.org/10.1145/1143844.1143874. https://doi.org/10.1145/1143844.1143874

[27] Searson, D.P.: GPTIPS 2: an open-source software platform for symbolic data mining. CoRR abs/1412.4690 (2014) https://arxiv.org/abs/1412. 4690 


\section{Biography of Authors}

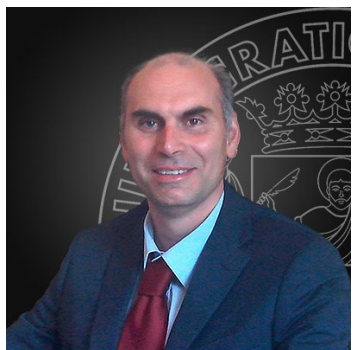

Gianni D'Angelo is a Tenured Adjunct Professor (RTD.B) at the University of Salerno, Italy. From April, 142021 he qualified as Second Level University Professor for the Sector 01/B1 - Computer Science, and from April 29, 2021 as Second Level University Professor for the Sector 09/H1 - Systems of Information Processing. He lectures "Computer Networks" and "Computer Architecture" at the Department of Computer Science of the University of Salerno. He has taught "Fundamentals of Computer Science and Programming" at the Department of Industrial Engineering of the same University. From 2012 to 2019, he has been a Contract Professor of "Elements of Computer Science" at the Department of Law, Economics, Management and Quantitative Methods of the University of Sannio, Benevento, Italy. He received the M.S. degree (cum laude) in Computer Engineering, and the Ph.D. degree in Computer Science, applied electromagnetism and telecommunications from the University of Salerno, Salerno, Italy, in 1998 and 2003, respectively. His research interests concern with the development and implementation of algorithms based on Artificial Intelligence, Deep Learning, and Machine Learning for Knowledge Discovery in BIG DATA context and implemented for High-Performance machines - HPC, and Parallel Computing. He gained experience in the world of the pattern recognition, neural networks, fuzzy logic, ANFIS systems, genetic and evolutionary algorithms, and parallel programming applied in various scientific and industrial fields. He authored many articles published in international journals, books and conferences, and currently serves as a reviewer, editorial board and guest editor for several international journals.

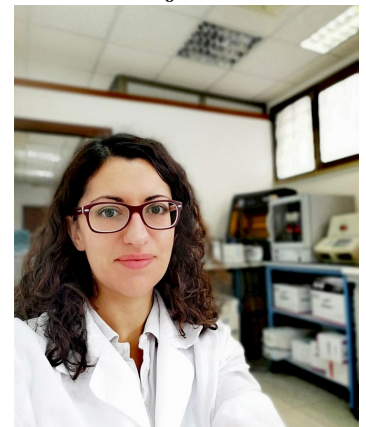

Maria Nunzia Scoppettuolo achieved the Bachelor's and the Master's Degree (cum laude) in Biology in 2005 and 2007, 
respectively, at the University of Sannio in Benevento, Italy. Then, she specialized in Medical Genetics in 2012, at the Department of Biochemistry and Medical Biotechnologies, University Federico II of Naples, Italy. In the last years, she was mainly interested in the study of different pathways involved in tumour progression in medulloblastoma, neuroblastoma and breast cancer and also clinical application of different therapeutic agents in the prevention of tumorigenesis and metastasis. Her expertise ranges from cell biology to molecular biology, with a remarkable competence in the field of Cytogenetics and Cytogenomic.

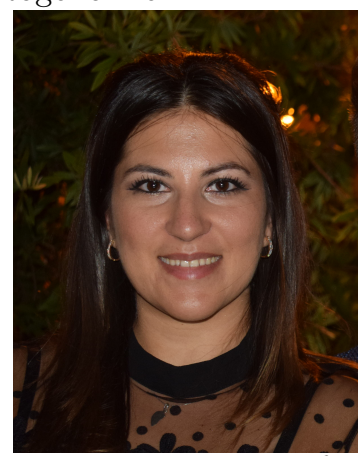

Anna Lisa Cammarota is a $\mathrm{PhD}$ student in Translational Medicine for Development and Active Aging at the Department of Medicine, Surgery and Dentistry "Scuola Medica Salernitana" of the University of Salerno, Italy. She obtained her MSc (cum laude) in Biology at the University of Salerno in 2020. She carried out research activities both as a trainee and as a research fellow from 2019 to 2021 at the laboratory of Biochemistry directed by Prof. Maria Caterina Turco, dealing with the study of tissue and serum biomarkers in both tumor and cardiovascular environment. Her work has contributed to the publication of two articles in international journals. Her current research project is focused on the study of the biological signature of pancreatic cancer, in particular on the development of early and preventive diagnostic methods through the use of artificial intelligence.

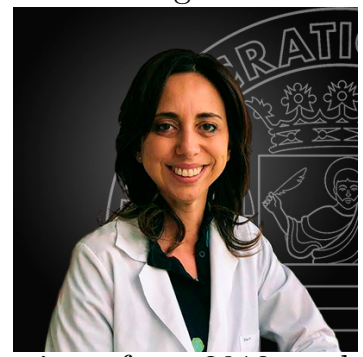

Alessandra Rosati is Associate Professor in Biochemistry from 2018 at the Departement of Medicine, Surgery and Dentistry of the University of Salerno, where she is presently active, both in education and research projects. In last years, she was mainly interested in the study of interactions between BAG3 and other proteins involved in the modulation of apoptosis (such as NF-kB, WT-1, Btk, and others) and in the development of antibodies for diagnostic and therapeutic uses. She is inventor of 5 patents in this field and is founding member of two academic spin-off. 


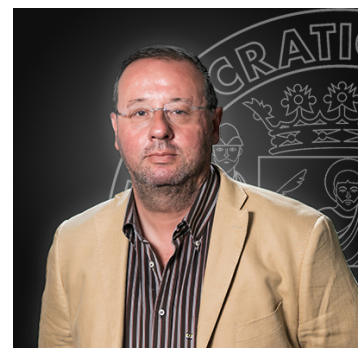

Francesco Palmieri is a full professor at the University of Salerno, Italy. He received from the same university an Italian M.S. "Laurea" degree and a $\mathrm{PhD}$ in computer science. His major research interests concern high performance networking protocols and architectures, routing algorithms and network security. Previously he has been an assistant professor at the Second University of Naples, and the Director of the telecommunication and networking division of the Federico II University, in Naples, Italy. At the start of his career, he also worked for several international companies on networking-related projects. He has been closely involved with the development of the Internet in Italy as a senior member of the Technical-Scientific Advisory Committee and of the CSIRT of the Italian NREN GARR. He has published a large number of papers (more than 200) in leading technical journals, books and conferences and currently serves as the editor-in-chief of an international journal and is part of the editorial board or associate editor of many other well reputed ones. 